\title{
Wales launches mouth cancer toolkit for dental teams
}

A new educational toolkit designed to help dental teams prevent and identify mouth cancer has been launched by Health Education and Improvement Wales (HEIW).

Dental teams are well placed to identify any abnormalities and play an important role in detecting cancers early during routine check-up appointments.

Kirstie Moons, Associate Director for Dental Team Workforce Planning and Development at HEIW, said: 'Our Mouth Cancer Quality Improvement Tool will help dental teams feel confident in providing preventative advice, diagnosing mouth cancers early and in supporting patients who have the disease'.

HEIW's new toolkit, which has been developed in collaboration with dental teams at Public Health Wales and Cardiff University, can be used by all members of the dental practice team - including dentists, dental nurses, dental hygienists and dental technicians to ensure an effective whole team approach to patient care.

The resource supports dental teams to: raise patient awareness of the causes of mouth cancer (including tobacco and alcohol use and the transmitting of the Human Papilloma Virus [HPV]); help patients become more aware of the symptoms; be confident in how to discuss suspected cancer with patients; and to be aware of available training and resources on the early detection of mouth cancer.

\section{BOOK REVIEW}

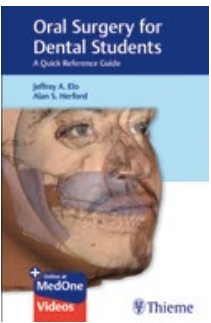

\section{ORAL SURGERY FOR DENTAL STUDENTS: A QUICK REFERENCE GUIDE}

Jeffrey A. Elo, Alan S. Herford;

2019; Thieme; $£ 49.99 ;$ pp. 264;

ISBN: 9781626239104

This 253-page book aims to provide readers with essential material in the basics of oral surgery. Although the title claims the book is for dental students, it can also act as a useful reference guide and revision tool for recent graduates and experienced practitioners. Written by Jeffrey Elo and Alan Herford, both professors of oral and maxillofacial surgery in California, the first four of 15 chapters cover introductory topics, including taking a history and examination of a new patient, infection control, preventing wrong site extraction, and anatomical considerations when injecting local anaesthetic.

Key areas in oral surgery are then covered, including odontogenic infections, interpreting radiographs, surgical extractions, antibiotic prescription, patients taking anticoagulant and antiplatelet medication, and managing medical emergencies. Clear headings are used throughout, with clear and concise bullet points in most sections. An evidence-based approach is followed, with each chapter including a bibliography for further reading.

Some readers will note the subtle but important differences between American and British recommendations regarding third molar removal, prophylaxis against infective endocarditis and antibiotic prescribing. For example, the authors suggest prescribing

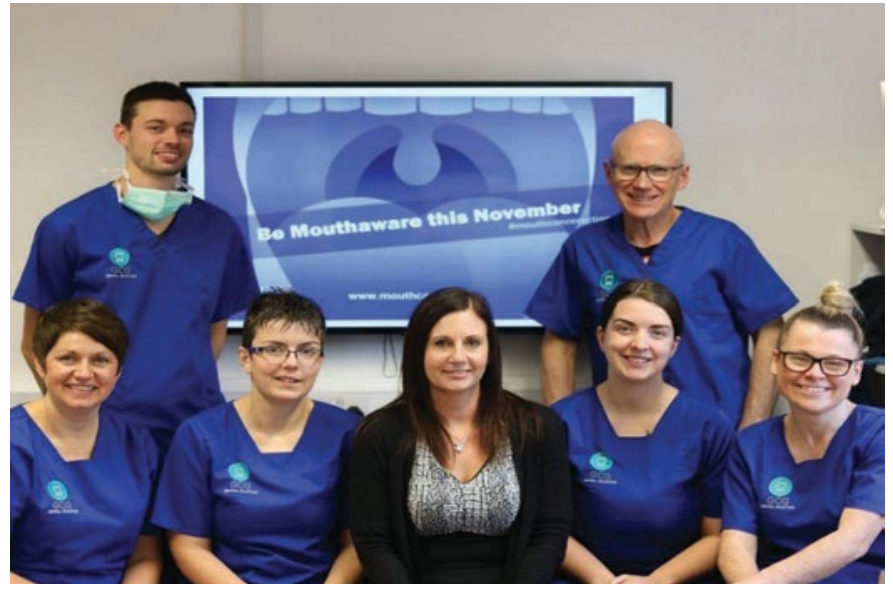

Gwaun Cae Gurwen Dental Practice team

Natalie Rees, a dental care professional involved in the pilot of the toolkit at Gwaun Cae Gurwen dental practice in Ammanford, said: 'Having recently seen a patient with mouth cancer, [the toolkit] allowed me to be confident in our practice processes'.

The Mouth Cancer Quality Improvement Tool was launched this November to coincide with Mouth Cancer Action Month.

a $2 \mathrm{~g}$ endocardititis prophylactic dose of amoxicillin, where SDCEP guidelines recommend $3 \mathrm{~g}$. They also advocate the prophylactic removal of wisdom teeth, which contradicts current NICE guidelines on wisdom tooth removal. For this reason, readers, especially dental students, should be cautious when referring to this book for clinical guidelines.

Whilst chapters on anatomy and common oral lesions are complemented by high quality images and diagrams, other chapters such as managing the pregnant patient and hypertension guidelines include extended bullet points with no visual aids to break up the text. Although I can appreciate that for certain subjects it may not be appropriate to use photos/diagrams, perhaps more summary boxes could have been used for readers to quickly reference.

Purchasers of this book will have access to the online e-book, as well as all the illustrations, and some educational videos. It is disappointing that there are only four videos, including an extraction on a patient and a suturing tutorial. More video content on different extraction techniques, performing a lymph node examination and raising flaps would certainly offer learners an improved learning experience.

In conclusion, Oral surgery for dental students will serve as a handy reference guide to dental students, recent graduates, and experienced practitioners. The book is easy to read and navigate through, with high quality illustrations utilised effectively to supplement the text. Whilst it is true that some of the clinical recommendations made by the authors are not consistent with UK national guidelines, this book will remain to serve as an excellent revision tool.

By Hassan-Ali Ismail 